\title{
Sophie Nizard (et al.), La table dressée : nourritures et identités chez les Juifs de France $\left(\mathrm{XIX}^{\mathrm{e}}-\mathrm{XX}^{\mathrm{e}}\right.$ siècles)
}

Peronnas, Les Belles Lettres, Archives juives. Revue d'histoire des Juifs de France, $n^{\circ} 47 / 1,1^{\text {er }}$ semestre 2014, 160 p.

\section{Régine Azria}

\section{(2) OpenEdition} Journals

Édition électronique

URL : http://journals.openedition.org/assr/26515

DOI : $10.4000 /$ assr.26515

ISSN : $1777-5825$

Éditeur

Éditions de l'EHESS

Édition imprimée

Date de publication : 31 décembre 2014

Pagination : 256

ISBN : 978-2-7132-2467-6

ISSN : 0335-5985

Référence électronique

Régine Azria, «Sophie Nizard (et al.), La table dressée : nourritures et identités chez les Juifs de France ( $x x^{\mathrm{e}}-\mathrm{xx}$ e siècles) », Archives de sciences sociales des religions [En ligne], 168 | 2014, mis en ligne le 18 mai 2015, consulté le 22 septembre 2020. URL : http://journals.openedition.org/assr/26515 ; DOI : https://doi.org/10.4000/assr.26515

Ce document a été généré automatiquement le 22 septembre 2020.

(C) Archives de sciences sociales des religions 


\section{Sophie Nizard (et al.), La table} dressée : nourritures et identités chez les Juifs de France ( $\mathrm{XIX}^{\mathrm{e}}-\mathrm{XX}^{\mathrm{e}}$ siècles)

Peronnas, Les Belles Lettres, Archives juives. Revue d'histoire des Juifs de France, $\mathrm{n}^{\mathrm{o}} 47 / 1,1^{\mathrm{er}}$ semestre 2014, $160 \mathrm{p}$.

\section{Régine Azria}

\section{RÉFÉRENCE}

Sophie Nizard (et al.), La table dressée : nourritures et identités chez les Juifs de France

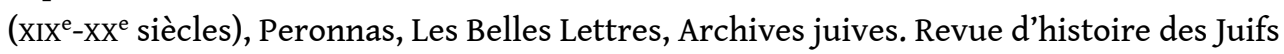
de France, $\mathrm{n}^{\circ} 47 / 1,1^{\text {er }}$ semestre 2014, $160 \mathrm{p}$. 
1 Catherine Nicault, qui signe l'éditorial de cette livraison de la revue Archives juives rappelle fort justement que «se nourrir est pour l'homme une nécessité, mais c'est toujours aussi un acte éminemment culturel ». Le titre du dossier ici proposé «La table dressée » en est la parfaite illustration dans la mesure où il suggère non seulement la mise en scène, mais aussi l'environnement et les conditions sociales et culturelles qui accompagnent l'acte de se nourrir. Si l'on ne mange pas n'importe quoi, on ne mange pas non plus n'importe comment ni avec n'importe qui. Chaque société, chaque culture édicte et impose ses façons de se nourrir. Ce en quoi la nourriture participe à l'identité d'un groupe. Ainsi,

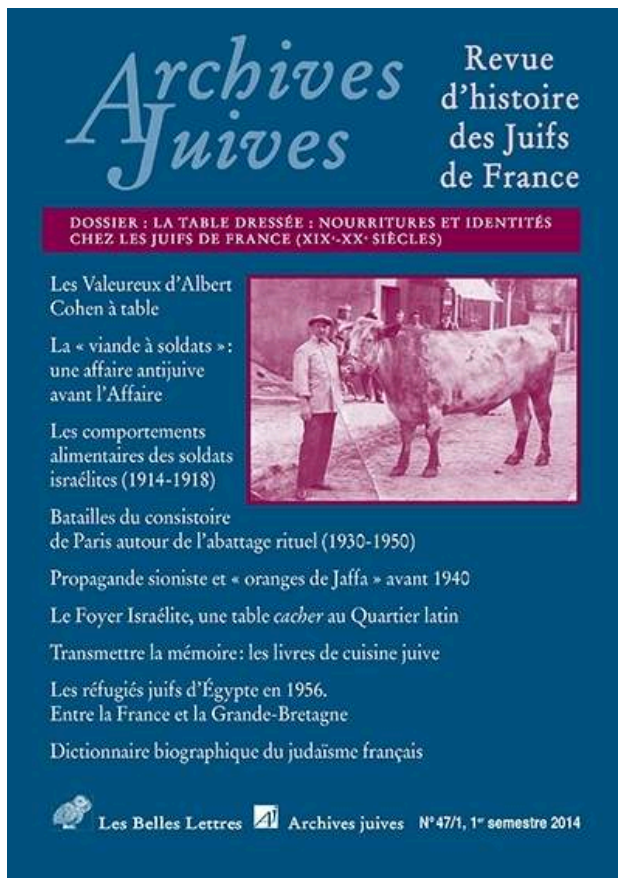
dans nos sociétés, il est/était de tradition de manger assis autour d'une table bien mise, d'où la table dressée. Mais dans sa version hébraïque - choulhan aroukh - cette formule évoque autre chose encore. Sophie Nizard le précise dès les premières lignes de son introduction: Choul'han Aroukh est le titre d'un code de lois écrit au XVI ${ }^{\mathrm{e}}$ siècle par Joseph Caro, un éminent talmudiste de Safed. Ce code, qui fait autorité aujourd'hui encore dans le monde juif pratiquant, traite des normes de conduite qui s'imposent dans les faits et gestes de la vie quotidienne, au nombre desquelles les normes alimentaires. Il n'est sans doute pas exagéré de suggérer que, bien plus qu'à son contenu, cet ouvrage de référence doit une part de sa notoriété auprès de milieux juifs sécularisés peu versés dans la littérature talmudique à son titre imagé. Et c'est probablement pour cette même raison - l'impact de la formule - que Joëlle Bahloul (ou son éditeur avisé) avaitchoisi d'intituler le livre tiré de son enquête sur les pratiques alimentaires des Juifs d'Algérie, Le Culte de la table dressée (Paris, Métailié, 1983). Cette étude demeure jusqu'à ce jour sans équivalent dans la littérature anthropologique francophone.

2 Dans le dossier dont il est question ici, le propos est quelque peu différent. Il s'agit, au dire de ses maîtres d'œuvre, d'interroger au long des sept contributions qui le composent la continuité du groupe à partir de la continuité de ses pratiques alimentaires, et ce dans des situations diverses, voire pour certaines extrêmes : guerre, Occupation, contextes migratoires.

3 Sans entrer dans le détail de chaque étude de cas, quelques remarques de fond, qui valent d'un article à l'autre, méritent une attention particulière. À commencer par la nécessaire prise en considération de la distinction entre respect des lois de la cacherout et continuité des pratiques culinaires, l'un n'étant ni l'équivalent ni la condition de l'autre. Le premier, qui concerne l'orthopraxie, relève de la sphère rituelle, religieuse et savante, tandis que la seconde a partie liée avec la culture et la transmission matérielles, lesquelles exigent un savoir-faire que l'on acquiert par la pratique plus que par l'étude des livres savants. Reprenons l'introduction de Sophie Nizard : « la tradition 
alimentaire est faite des deux : d'une part rites et pratiques, normes et écarts à la norme, lois inscrites et discutées dans les livres fondateurs - Torah, Talmud, codes légaux - dont l'étude a été longtemps inaccessible aux femmes; d'autre part, cuisine élaborée et transmise de mères en filles ", écrit-elle (p. 6). Or, dans le cas des pratiques effectives, c'est précisément l'écart à la norme et le discours qui accompagne cet écart et le justifie parfois, qui retiennent l'attention. Quant au respect de la cacherout, il peut tout aussi bien s'appliquer à des plats et recettes non traditionnels, dès lors que ces derniers s'avèrent « compatibles » au regard de la norme religieuse.

Dans sa contribution à ce dossier (« Entre fidélité culturelle et transgression rituelle, les nourritures dans l'œuvre d'Albert Cohen», p. 11-27), Claudine Nacache-Ruimi nous livre deux exemples particulièrement «savoureux » de ces écarts. C'est Mangeclous, le héros gargantuesque imaginé par Albert Cohen, qui le lui fournit. Répondant à son comparse Salomon épouvanté à l'idée que son ami consente à manger du porc, Mangeclous affirme avec la plus parfaite mauvaise foi que « le jambon est la partie juive du porc ». (Albert Cohen, Les Valeureux, Paris, Gallimard, Folio, 1969, p. 253). Resituée dans le contexte de l'univers d'Albert Cohen et du petit monde haut en couleur des Valeureux, cette réplique hilarante, qui entend justifier l'acte de transgression qu'il est sur le point de commettre, montre à l'évidence qu'il n'y a pas là volonté d'assimilation ni même volonté de transgression, mais plus fondamentalement désir d'accommodation. Pour preuve, mais a contrario, cet autre passage du même roman dans lequel «la création de la moussaka " par le même personnage, donne lieu à un morceau de bravoure. À l'intention des lecteurs qui n'auraient pas la chance de connaître la moussaka, il est bon de préciser au préalable qu'il s'agit d'un plat grec dans la composition duquel entrent, outre aubergines, tomates et oignons, de la viande hachée (bœuf ou agneau) et de la sauce béchamel faite à partir de lait, de beurre et de farine. On peut aussi peaufiner en recouvrant de fromage râpé afin d'obtenir un plat gratiné. Or le mélange lacté/carné est formellement interdit par les lois de la cacherout. Qu'à cela ne tienne! Les Juifs grecs et thessaloniciens amateurs de moussaka qui n'entendaient pas renoncer à s'en régaler, dont notre Mangeclous, se sont fait fort d'adapter la recette, autrement dit de la "cachériser ", en supprimant béchamel et fromage râpé. D'où cette remarque de Claudine Nacache-Ruimi : « Du côté de l'écriture, l'auteur [Albert Cohen] montre combien sont infinies les ressources de la langue quand celle-ci se pique au jeu de l'enchantement. Sur le plan des idées, il rappelle quelques grands noms, quelques grands principes, et réaffirme sa double appartenance : héritier de la parole de la Bible et dépositaire de la culture française, Cohen ne manque jamais de revendiquer, même sur le mode ludique, les deux courants qui circulent dans son œuvre. Quant à la moussaka... elle demeure conforme aux prescriptions énoncées dans l'Ancien Testament ». Ce désir d'adaptation, on le retrouvera en bien d'autres occasions, à table et hors de la table. Cela étant, si l'écart à la norme est ici le fait d'un choix quelque peu provocateur pleinement assumé, il peut aussi dans d'autres circonstances être contraint.

5 Une situation qu'analyse Philippe Landau à propos des comportements alimentaires des "poilus » juifs de la guerre de 14-18 («Les comportements alimentaires des "poilus" juifs. Le reflet de l'identité franco-israélite», p. 37-56). Partant du constat d'une désagrégation- relative - des pratiques ancestrales juives dans la France républicaine et laïque du début $d u x^{e}$ siècle, l'auteur s'intéresse au comportement alimentaire des quelque 36000 Juifs de France et d'Algérie mobilisés à la déclaration de la guerre, notamment de ceux demeurés attachés aux pratiques traditionnelles. Si le respect du 
chabbat leur est évidemment impossible, qu'en est-il de celui de la cacherout? De fait, nous apprend P. Landau, «les autorités françaises ont réglé dès 1807 la question de l'observance des lois religieuses par les conscrits et les soldats en temps de guerre avec les responsables de la communauté juive ». Cela s'est fait dans le cadre du Grand Sanhédrin réuni par Napoléon $1^{\mathrm{er}}$. En réponse aux questions relatives à la capacité du judaïsme à concilier les préceptes de la Torah et les articles du Code civil, les rabbins émettent l'avis suivant : «tout israélite appelé au service militaire est dispensé par la Loi [religieuse], pendant la durée de ce service, de toutes les observances religieuses qui ne peuvent pas se concilier avec lui ». Cette tolérance, ajoute P. Landau, est en réalité une entorse à la législation juive qui ne sera jamais remise en cause par le Consistoire central. Soucieux de donner des gages de patriotisme, les responsables consistoriaux s'efforcent en effet de persuader leurs ouailles que «le devoir militaire est, avec l'acquisition d'une instruction moderne, un des moyens de faire l'apprentissage de la citoyenneté ». Mais qu'en est-il du côté des intéressés eux-mêmes? Sont-ils prêts à sacrifier leurs convictions religieuses sur l'autel du patriotisme? On dispose de peu de témoignages de combattants juifs sur la question concède Philippe Landau... Quant aux correspondances disponibles, elles semblent confirmer le fait que les hommes condamnés à supporter la faim et le froid dans la boue des tranchées ont d'autres préoccupations. Même s'il subsiste une sensibilité religieuse ou communautaire à l'approche des fêtes, la plupart ne se soucient guère du chabbat ou de la cacherout. La vie est dure au front, la nourriture y est de piètre qualité, insuffisante, le plus souvent consommée dans des conditions rudimentaires. Avant de songer à manger cacher, on se préoccupe de s'alimenter tout simplement, pour tenir. Très vite les réticences tombent face aux nourritures interdites, car la faim est omniprésente. Certains pourtant tentent d'éviter ces « écarts à la norme ». Ils comptent sur les colis qu'ils reçoivent de l'arrière ou échangent leur gamelle contre du pain, du sucre, des œufs ou des biscuits. Les Juifs d'Algérie, nombreux dans les régiments de zouaves, sont les plus attachés à l'observance des règles de la cacherout, dans les limites du possible : «dans l'incapacité de préserver toutes leurs traditions, dans ce cadre, ils cherchaient néanmoins à en respecter l'essentiel, refusant de manger du porc et de mélanger la viande avec le laitage, mais acceptant de consommer du bœuf et du poulet qui n'avaient pas été abattus rituellement» (p.46). C'est à l'approche de la fête de pessah, symbole de la libération de l'esclavage, que les combattants juifs, agnostiques ou croyants, ressentent de façon plus intense leur judéité. Faute de pouvoir se réunir en famille pour le repas traditionnel du seder, ils s'organisent, avec ou sans l'aide des aumôniers militaires, pour se procurer des matzot (pains azymes) et célébrer en commun la sortie d'Égypte. Sentiment d'appartenance plus que conformité à la loi religieuse sans doute, que les circonstances extrêmes incitent à réaffirmer.

Ce n'est plus d'écarts à la norme mais bien de pratiques transgressives qu'il est question dans la contribution de Jean Laloum (Le Consistoire de Paris et les commerces de bouche : l'enjeu de l'abattage rituel [années 1930-1950], p. 57-78). «À la fin des années 1920, écrit l'auteur, de plus en plus de restaurants affichent faussement l'estampille cacher (en lettres hébraïques) sur leurs devantures, et nombreux sont les consommateurs qui se prennent à douter de la conformité rituelle de la nourriture qui leur est proposée. Chargé de veiller à l'observance de la cacherout dans la communauté, le Consistoire - et plus particulièrement l'autorité rabbinique - est régulièrement interpellé sur des pratiques complaisantes, voire frauduleuses tant par les fidèles que par ses propres services. Tout le circuit de production et de commercialisation de la 
viande et de ses dérivés [est] incriminé ». Les principaux acteurs concernés sont les "sacrificateurs " en charge de l'abattage rituel et de la vérification de l'état sanitaire des animaux sur pieds puis des carcasses, les bouchers (gros et détails), les charcutiers, les surveillants désignés par l'autorité cultuelle, le grand rabbin de Paris dont le cachet de certification apposé sur chaque morceau de viande porte le nom. Or ce système présente de nombreuses failles, tricheries et tromperies dont les autorités consistoriales sont activement ou passivement complices. Finalement, des produits non cachers sont frauduleusement estampillés cachers et mis en vente dans des commerces supposés délivrer des produits rituellement conformes. Le pragmatisme économique des intervenants concernés explique pour partie ces « dysfonctionnements », le surcoût occasionné par les contraintes qu'impose le consistoire (taxes, salaires des surveillants, limitation des jours d'ouverture, etc.) incitant les acteurs économiques soit à sortir du système, soit à tricher. Le consistoire doit imposer des sanctions suffisamment dissuasives pour venir à bout de ces pratiques frauduleuses récurrentes. Il hésite néanmoins à recourir à la justice civile. Ce tableau déjà passablement compliqué s'assombrit encore avec l'arrivée des Juifs immigrés. Ceux-ci, rituellement plus rigoristes, hostiles à tout accommodement tel que l'autorisation d'ouverture des commerces le samedi, dénoncent le laxisme, l'incompétence et les dérives, ils contestent le monopole consistorial sur la cacherout, et créent de leur côté leur propre circuit de cacherout labellisé par leurs propres rabbins. Sous l'occupation enfin le consistoire se trouve confronté à une situation plus dramatique encore avec la mise en place des lois antijuives et l'interdiction faite aux Juifs d'exercer certaines professions. $\mathrm{Au}$ terme de moult démarches, une ordonnance allemande prévoit néanmoins le maintien de deux catégories d'entreprises juives, celles dites strictement " rituéliques ", c'est-à-dire délivrant une alimentation cachère, et celles appelées «boutiques de ghetto» destinées à approvisionner les populations juives non pratiquantes.

7 Jusqu'en 1967, le restaurant de la rue de Médicis à Paris est le seul à proposer de la nourriture cachère aux étudiants du Quartier Latin. Ouvert pendant l'entre-deuxguerres à l'intention d'une clientèle majoritairement achkenaze, le Foyer israélite devient après sa réouverture dans les années 1950, le lieu obligé des étudiants juifs venus d'Afrique du Nord désireux de manger cacher (Martin Messika, « une table cacher et conviviale au Quartier Latin: le Foyer israélite dans les années 1950 et 1960 », p. 103-112). De fait, la qualité des plats est médiocre : « les restau U n'ont jamais eu une réputation de relais gastronomiques » dira un des informateurs, les menus n'ont rien de traditionnel ou rituel, à quelques exceptions près (couscous ou cholent). À cet égard, ils manifestent une forme d'ouverture culturelle: bœuf mode et mironton sont fréquemment à la carte, parfois du goulash hongrois. Ainsi se trouvent dissociés respect de la cacherout et continuité des cuisines traditionnelles spécifiques : «Le restaurant universitaire constitue ainsi un espace dans lequel manger cacher n'est plus lié à des spécialités juives » constate l'auteur. Ceux qui ne sont pas tenus par la cacherout y viennent surtout pour y retrouver des connaissances de leur pays d'origine et se ressourcer dans une ambiance familière. Se profile ainsi la fonction sociale et identitaire de ce lieu qui sera la cible de terroristes, poseurs de bombe en 1979.

8 D'antisémitisme il n'a pas encore été question dans cette recension. Il est notoire pourtant que les pratiques alimentaires des Juifs ont suscité bien des fantasmes propres à exacerber l'antijudaïsme traditionnel à commencer par l'accusation de crimes rituels. Comme le démontre l'affaire de la "viande à soldats », les rumeurs les plus infondées 
peuvent à leur tour devenir prétexte à l'antisémitisme moderne (Grégoire Kauffmann, "L'affaire de la "viande à soldats". Une campagne antisémite en 1892 », p. 28-36). C'est le marquis de Morès, un aventurier désargenté proche d'Édouard Drumont (fondateur du journal antisémite $L a$ Libre Parole, auteur de La France juive, 1886), qui lance l'affaire à partir d'une rumeur concernant deux bouchers juifs de Verdun. Affirmant avoir mené lui-même sa propre enquête, il les accuse d'avoir acheté sous la Grande Halle de la Villette, une quarantaine de bêtes impropres à la consommation, "charognes ambulantes ", "malsaines ", "dans un état absolument dégoutant ", dont la viande aurait été distribuée aux soldats de la garnison. À la suite d'une plainte déposée auprès du Procureur de la République, ce dernier ordonne une enquête. Elle révèle qu'en effet la qualité des animaux laisse à désirer mais que les viandes débitées par les bouchers incriminés «sont toutes consommables ... et que les soldats de Verdun n'ont jamais exprimé la moindre doléance à ce sujet. ». Cette affaire de la "viande à soldats » aura des suites puisque Drumont n'hésitera pas à suggérer l'existence d'un lien direct avec l'accusation d'espionnage visant le capitaine Dreyfus: «si les Juifs, par haine de la France, sont capables d'empoisonner l'armée, c'est qu'ils sont aussi capables de la trahir ».

Enfin, Valérie Assan et Sophie Nizard referment ce dossier avec un article dont le titre proustien - «Les livres de cuisine juive: à la recherche d'un monde perdu?» (p. 113-131) - nous invite à nous interroger sur les causes du succès que connaît ce type d'ouvrages, en particulier depuis les années 1990. Si plus du tiers de ces publications font explicitement référence à un espace géographique ou culturel spécifique (Afrique du Nord, Europe orientale, Alsace, Grèce, etc.), signe de la présence dans notre pays de Juifs venus d'horizons divers, la plupart des livres se présentent comme des ouvrages de «cuisine juive » et proposent un éventail de traditions culinaires, tour du monde culinaire en quelque sorte. À ceux-là s'ajoutent les livres qui se soucient moins de l'origine des recettes que du respect des prescriptions religieuses, étant entendu par ailleurs qu'aucun des livres de cuisine juive recensés par les auteurs ne fait entrer d'aliments ou de mélanges interdits dans les recettes proposées. Il y en a ainsi pour tous les goûts et tous les types de publics. Mais encore? S'interrogeant sur les causes de cet engouement, nos deux auteurs inscrivent leur réflexion dans un contexte plus large et suggèrent que le succès croissant de la littérature culinaire (y incluse la cuisine juive) serait lié au mouvement global actuel d'affirmations identitaires dont la cuisine serait une des manifestations parmi d'autres. On assisterait ainsi à la patrimonialisation des traditions culinaires nationales, régionales et autres et à la nécessité que représenterait leur transmission face au risque de perte et d'oubli. Passée cette première étape de la réflexion vient immanquablement la question : que s'agit-il de transmettre? Plusieurs réponses destinées aux différents types de publics concernés. S'adressant à « la jeune femme juive moderne qui tout en gardant ses traditions [sous-entendu la cacherout] n'en a pas moins envie de goûter les plats du pays dans lequel elle vit » et n'est pas insensible aux normes de la diététique moderne, l'éditeur de tel livre propose non pas « un livre de cuisine juive, mais un livre de cuisine française adaptée aux lois de la cacherout»(et de la diététique). Se succèdent astuces et conseils, suggestions de produits de substitution aux produits et/ou mélanges interdits. Nous ne sommes pas loin ici de la moussaka compatible de Mangeclous que nous avons rencontrée précédemment.

10 Cela étant, une autre caractéristique, et non des moindres, de ces livres est leur fonction mémorielle. La table dressée est un lieu de mémoires composite : mémoire des 
lieux qu'on a quittés et d'un passé qui n'est plus, mémoire des odeurs et des saveurs des marchés et des cuisines de l'enfance, mémoire des sons et des mots propres à la cuisine, mémoire du calendrier des fêtes juives: à chaque fête son menu traditionnel et les effluves qui vont avec. Mais menu qui diffère d'un point du globe à l'autre. L'espace de la cuisine est propice au récit, et la confection des recettes de jadis libère la parole. Les préfaces des livres de cuisine juive en témoignent, leurs auteurs jugeant parfois nécessaire de raconter, d'expliquer, de décrire la vie de jadis lorsque ces plats s'imposaient à l'évidence. Constat - connu d'avance ? - de nos auteurs : «L'ambition de la plupart des auteurs de livres de cuisine juive dépasse assurément le cadre culinaire. Il ne s'agit pas seulement d'expliquer la bonne technique ni d'indiquer les justes proportions [l'unité de mesure étant plus souvent le creux de la main que la balance ou le verre gradué], mais de restituer des saveurs, des parfums et des couleurs; plus encore : des émotions, des lieux, un mode de vie, tout un monde défunt ». Ce faisant, dans leur collecte de recettes, les auteurs de ces livres se font ethnographes, «tels les folkloristes recueillant les contes populaires transmis par la tradition orale [... ils] ont conscience de transcrire pour la première fois ce qui était jusque-là livré exclusivement par la parole et par le geste ».

11 Ce dernier point me suggère une remarque : la mise par écrit de la tradition orale de commentaires rabbiniques de la Torah écrite, qui sera à l'origine du Talmud, fut décidée pour éviter la perte et l'oubli de tout un patrimoine spirituel et intellectuel au lendemain de la destruction du second Temple. La mise par écrit de recettes et de traditions culinaires jusque-là transmises par la parole et le geste s'opère pour des motifs du même ordre: dispersion des communautés, rupture des chaînes de transmission, transformation des pratiques alimentaires. La logique est identique. Cela étant, l'irruption d'internet et des réseaux sociaux évoqués en fin d'article change probablement la donne.

12 Combien riche et varié, ce thème de l'alliance entre les nourritures et les identités. Ce dossier pourtant fourni est loin d'en avoir épuisé tous les aspects. Gageons qu'il saura aiguiser la curiosité de nos jeunes collègues et leur envie de poursuivre la réflexion ici engagée. À cette table dressée, conviviale et accueillante sont conviés chercheurs de tous terrains et spécialistes des cuisines du monde pour un joyeux festin interdisciplinaire. 